Published in Langmuir (2008) 24(14):7580-7589

\title{
Heterogeneous Drying of Colloidal Polymer Films: Dependence on Added Salt
}

Alexander M. König, ${ }^{1}$ Tecla G. Weerakkody, ${ }^{2}$ Joseph L. Keddie ${ }^{*}{ }^{2}$ Diethelm Johannsmann ${ }^{* 1}$

${ }^{1}$ Institute of Physical Chemistry, Clausthal University of Technology, Arnold-Sommerfeld-Str. 4, D-38678 Clausthal-Zellerfeld, Germany

${ }^{2}$ Department of Physics, Faculty of Engineering and Physical Sciences, University of Surrey, Guildford, Surrey, GU2 7XH, UK

\begin{abstract}
Using magnetic resonance profiling coupled with dynamic light scattering, we have investigated the mechanisms leading to the formation of a partly coalesced surface layer, or "open skin", during film formation from waterborne polymer dispersions. We present the first use of the skewness of the distribution of free water as a model-free indicator of the spatial non-uniformity of drying. The skewness reaches a maximum at the same time at which a strong, static component - presumably originating from a skin at the film/air interface - appears in the light scattering data. The average solids content at this time is around 53 vol.\%. Addition of salt to the dispersion increases both the skewness of the distribution of free water and the propensity for skin formation. Surprisingly, the drying is influenced not only by the concentration and valency of the ions in the salt but also by the particular ion. At intermediate particle densities, added salt strongly lowers the cooperative diffusion coefficient, $D_{\text {coop}}$. When the particles reach close packing, $D_{\text {coop }}$ sharply increases. If the particles readily coalesce, the effects of the increased diffusivity will be counteracted, thereby inducing the formation of a skin. A modified Peclet number, $P e$, using $D_{\text {coop }}$, is proposed, so that the presence of salt is explicitly considered. This modified $P e$ is able to predict the non-uniformity in
\end{abstract}

\footnotetext{
* authors for correspondence:

electronic mail: johannsmann@pc.tu-clausthal.de, phone +49 5323 - 72 3768, fax : +49 5323 - 724835

electronic mail: j.keddie@surrey.ac.uk, phone +44 1483 - 686803, fax +44 1483 - 686781
} 
Published in Langmuir (2008) 24(14):7580-7589

drying that leads to skin formation. 
Published in Langmuir (2008) 24(14):7580-7589

\section{Introduction}

The formation of polymer films from waterborne polymer dispersions is a process of everincreasing practical relevance. ${ }^{1,2,3}$ A major driving factor certainly is the compliance with environmental regulations, which severely limit the amount of volatile organic compounds (VOCs) that can be released into the atmosphere upon drying. Film formation is commonly divided into three stages, which are the evaporation of water leading to particle packing (stage I), particle deformation to fill all available volume (stage II), and interdiffusion across particle/particle boundaries, i.e., coalescence (stage III). During the first stage, water evaporates until the particles come into contact. Subsequently, the particles deform as the interstitial water is eliminated. Finally, any surfactant membranes break so that polymer chains from neighboring particles interdiffuse, thereby producing a homogeneous, tough film.

As has been frequently emphasized, film formation usually proceeds in a spatially heterogeneous way. ${ }^{1}$ Heterogeneity occurs both in the plane of the film ${ }^{4,5}$ (i.e. edge-in drying) and perpendicularly to the substrate ${ }^{6,7}$ (i.e. top-down drying). The time at which water recedes from a film's edge has been demonstrated to be proportional to the reduced capillary pressure. ${ }^{6}$ Edge-in drying can be prevented by blowing hot air across the film surface. Vertical gradients in water concentration can never be entirely avoided because these gradients drive the transport of water towards the film surface. However, the extent and consequences of vertical gradients in water concentration vary considerably. If particles are packed together at the film/air interface, and if the particles are subject to wet sintering (being highly deformable), then a process model developed by Routh and Russel (R-R model) predicts coalescence to create a so-called "skin" layer above a wet layer. ${ }^{6,8}$ There is clear experimental evidence for the development of skin layers. ${ }^{9,10}$ 
In applications, skin formation is usually considered to be a serious problem. Water transport by diffusion through a coalesced polymer layer is slower than flow along particle/particle boundaries. ${ }^{11}$ Experiments have shown that a skin layer on a latex film traps water beneath it, thereby slowing down the drying process. ${ }^{12}$ Subsequently, trapped water will reduce the film's mechanical strength and adhesion. ${ }^{13}$ Furthermore, as drying proceeds, the skin might rupture or wrinkle, which will result in an irregular surface. ${ }^{14}$ For these various reasons, the topic has attracted intense interest.

Simulations predict that colloidal particles will accumulate at the surface of thick films undergoing relatively fast drying. ${ }^{15}$ Cryogenic SEM images of films that were quenched during the process of drying have provided impressive evidence to support this view. ${ }^{16}$ In these images, one can clearly see particles being accumulated at the top of the film. Metaphorically speaking, the airwater interface acts like a snow plough, piling up particles just below the phase boundary. Pile-up is prevented if the recently-created concentration gradients quickly equilibrate via diffusion. The competition of pile-up and diffusion has been put into quantitative terms in a dimensionless parameter, called the Peclet number, $P e{ }^{6,8} P e$ is the ratio of two characteristic time scales, which are the time needed to diffusively equilibrate gradients in particle concentration and the time of drying. For a film of initial thickness, $H_{0}$, with water evaporation leading to the surface receding at a speed, $E, P e$ can be most simply expressed as $H_{0} E / D_{0}$, where $D_{0}$ is the Stokes-Einstein diffusion coefficient. Particle accumulation occurs whenever the speed of drying exceeds the rate by which concentration gradients decay. This condition corresponds to $P e$ 1. Recent experimental evidence $^{17}$ shows that the water concentration gradient in the vertical direction is proportional to $P e^{0.8}$, which compares to a prediction of $P e^{0.5}[15]$. Note, however, that the Stokes-Einstein law only holds in the dilute limit. A drawback of basing the calculation of $P e$ on the Stokes-Einstein diffusivity is that this equation does not account for particle/particle interactions. 
A second prerequisite for skin formation within the R-R model is a sufficiently high deformability of the particles, so that they can form a continuous layer while the material underneath is still wet. More specifically, there is a second dimensionless number, $\bar{\lambda}$, which compares the time scale of particle deformation to the time scale of drying. One has $\bar{\lambda}=\eta_{0} a E /\left(\gamma_{\text {wat }} H_{0}\right)$ with $\eta_{0}$ the zero shear-rate viscosity of the polymer particles, $a$ the particle radius, and $\gamma_{\text {wat }}$ the energy of the air-water interface. For $P e>1$ but $\bar{\lambda} \quad 1$, particles will be in close contact at the film surface, so that they are elastically coupled, but they will not be coalesced. A so-called "crust" will be formed, as illustrated in Fig. 1.

In order to explore skin formation in colloidal dispersions, we have simultaneously applied magnetic resonance profiling (MRP) $)^{18,19}$ and diffusion wave spectroscopy (DWS $)^{20}$ to drying polymer films. There have been limited previous applications of light scattering techniques to studies of film formation, ${ }^{21,22}$ and there are no previous reports of MRP and DWS being used together in such a study. MRP yields the volume fraction of mobile water as a function of the distance from the substrate, $\varphi_{w}(z)$. Skin or crust formation is thereby indicated by a step in $\varphi_{w}(z)$ along the vertical direction, corresponding to a higher polymer concentration near the surface. DWS yields the mobility of particles inside the film. Given the complicated geometry in experiments on drying films, the particle self-diffusion coefficient cannot be quantitatively derived from the autocorrelation function. Still, one can compare different data sets and determine any differences in particle mobility. In addition to the particle mobility, light scattering contains an essential second bit of information, which is the amplitude of the autocorrelation function (ACF). Should a skin form at the top of a film, it will act as a static scatterer. This strong background is superimposed onto the dynamic fluctuations of scattering from the bulk that is still mobile at this time, which lowers the amplitude of the ACF. 
A previous study by Erkselius et al. ${ }^{12}$ has provided the first significant evidence that electrostatic stabilization influences the drying rate and total drying time of latex films. In our research reported herein, salt was added to the dispersions in order to vary the interparticle forces. The influence of salt is threefold: Firstly, salt screens the electrostatic repulsion, thereby widening the local cages of confinement around the particles and allowing for faster Brownian motion. The short-time selfdiffusion coefficient of the particles increases. Secondly, electrostatic screening lowers the osmotic pressure of the particles and thereby the thermodynamic driving force for the equilibration of concentration fluctuations. Thirdly and importantly, salt can induce particle aggregation ("saltingout") in the late stages of drying by reducing the barrier to aggregation. ${ }^{23}$ In Ref. 12 , faster drying has been attributed to the presence of salt, when particle flocculation at higher ionic concentrations caused sedimentation and the avoidance of a skin layer. ${ }^{12}$

Regarding the second point, the evolution of a concentration profile in colloidal dispersions is governed by the cooperative diffusion coefficient, $D_{\text {coop }}$ (also called "mutual diffusion coefficient"). The relation between $D_{\text {coop }}$ and $D_{0}$ (valid at infinite dilution) can be rather complex. We provide an estimate in the Discussions section. When particles strongly repel each other (for instance, due to electrostatic interaction) the equilibration of concentration gradients speeds up considerably because of cooperative motion. $D_{\text {coop }}$ must not be confused with the self-diffusion coefficient, which governs Brownian motion. In situations where individual particles cannot leave the local cages because the packing is too high, cooperative motion along the concentration gradients will still occur. At moderate concentrations, where the particles interact, but are not yet close-packed, $D_{\text {coop }}$ is much greater than $D_{0}$ if the particle surfaces are charged. The effect of charge on $D_{\text {coop }}$ is reduced by the addition of salt in the continuous medium. 
It is also relevant to note that the tendency of ions to "salt-out" colloidal particles is poorly understood. The tendency for different ions to influence colloidal stability can be placed in a welldefined order called the Hofmeister series. ${ }^{24,25}$ The Hofmeister series cannot be explained merely by electrostatic screening as described by the DLVO theory, which considers the valency of the ions. Numerous properties of electrolyte solutions (including protein aggregation, the speed of chemical reactions, catalytic activity of enzymes, surface tension, the critical micelle concentration, and others) depend on the type of the dissolved ions, and the dependency follows the order of the Hofmeister series. ${ }^{25}$ The fact that so many diverse properties all lead to the Hofmeister series suggests that there is a general mechanism underlying these different manifestations. The operative mechanism is still under debate. The ion's tendency to stabilize or destabilize the water structure around it has been regarded as a key factor. Recently, however, this notion has been questioned ${ }^{26,27}$ and dispersion forces have been put forward as an alternative explanation. ${ }^{28}$

A recent study ${ }^{27}$ has shown that the order of the ions in the Hofmeister series can be correlated with their tendency to disorder alkyl chains in fatty acid layers at interfaces. We speculate that Hofmesiter effects could also contribute to the de-stabilization of surfactant bilayers at particle/particle interfaces. We note that the R-R model ${ }^{8}$ assumes that any surfactant membranes readily rupture when the particles come into contact, and the effects of salt are not considered. Hence, ion effects are not predicted by the model and could yield otherwise unexplainable results.

\section{Materials}

An acrylic latex was prepared by miniemulsion polymerization. A copolymer was polymerized from butyl acrylate and methyl methacrylate in a 1:1 weight ratio, using an anionic surfactant (Dowfax 2A1). A mixture of ammonium persulfate and sodium meta bisulfate was used as the initiator. These initiators impart a negative charge to the particle surfaces, which adds to the charge 
of the surfactant. The average particle diameter, according to dynamic light scattering measurements, was $180 \mathrm{~nm}$ and the initial solids content was $47 \mathrm{wt} \%$. The glass transition temperature was determined to be $T_{g} \sim 18^{\circ} \mathrm{C}$ by differential scanning calorimetry. Using the densities of $\rho_{\mathrm{PMMA}}=1.19 \mathrm{~g} / \mathrm{cm}^{3}$ and $\rho_{\mathrm{PBA}}=1.03 \mathrm{~g} / \mathrm{cm}^{3}$, one derives an initial solids content by volume of $\Phi_{0}=43 \mathrm{vol} . \%$. The addition of excess $\mathrm{NaCl}$ to the latex resulted in coagulation, indicating that the latex was charge stabilized.

Salt solutions were freshly prepared from the following salts: $\mathrm{Na}_{2} \mathrm{SO}_{4}, \mathrm{NaNO}_{3}, \mathrm{NaClO}_{4}$, and $\mathrm{MgCl}_{2}$. In order to have the same solids content in all experiments, a fixed volume of $1 \mathrm{~mL}$ of the particular salt solution was added to a $5 \mathrm{~mL}$ volume of the dispersion in each experiment. We chose two ionic strengths in the aqueous phase of the dispersion: $238 \mathrm{mmol} / \mathrm{L}$ and $119 \mathrm{mmol} / \mathrm{L}$. After dilution with the salt solution, $\Phi_{0}$ was 36 vol. $\%$. It is relevant to note that the dispersions containing $\mathrm{MgCl}_{2}$ at an ionic strength of $119 \mathrm{mmol} / \mathrm{L}$ were marginally stable. All other dispersions showed good stability.

\section{Magnetic Resonance Profiling}

Magnetic resonance profiling and its application to the drying of polymer dispersions have been described in the literature. ${ }^{7,18}$ Briefly, the drying film is situated in a magnetic field gradient that is normal to plane of the substrate (Fig. 2). The local field strength encodes the height above the substrate, and NMR signals can be separately acquired in horizontal cuts through the depth of the sample.

Wet films were cast onto clean glass cover slips $(2 \mathrm{~cm} \times 2 \mathrm{~cm})$ and immediately placed in the magnet at a position corresponding to a magnetic field strength of $0.7 \mathrm{~T}$ and a field gradient strength

of $17.5 \mathrm{~T} \mathrm{~m}^{-1}$. The initial film thickness, $H_{0}$, was typically $600 \mu \mathrm{m}$. The temperature within the 
magnet was $c a .25{ }^{\circ} \mathrm{C}$ for all experiments, and the air was static. Signals were obtained using a quadrature echo sequence $^{19}: 90_{x}-\tau-\left(90_{y}-\tau \text {-echo- } \tau-\right)_{n}$ for $n=32$ echoes and a pulse gap of $\tau=95.0 \mu \mathrm{s}$. To obtain a profile, the echoes were Fourier-transformed and then summed, thus giving an NMR signal intensity profile as a function of vertical position. No significant signal is observed from the polymer phase, and the water spin-spin relaxation time is largely independent of the polymer concentration in the dispersion. Hence, the NMR intensity is a fair measure of the water content that can be calibrated from the time-zero data with a known, uniform concentration. The pixel resolution in these experiments was about $15 \mu \mathrm{m}$. Due to its reliance on the spin-spin relaxation time, the instrument only probes free water. A film, which is dry in the sense of MRP, may still contain bound water. With regard to skin formation, free water is a more relevant parameter than the total amount of water because free water allows for fast mass transport and reflects the volume fraction of interstitial voids.

Fig. 3 shows three sets of water distribution profiles. The experiment shown in Fig. 3a was conducted on a dispersion with no added salt. The NMR signal intensity does not vary much with vertical position in the film, indicating that the distribution of water is rather homogeneous throughout the entire drying process. In the experiment shown in Fig. $3 \mathrm{~b}, \mathrm{MgCl}_{2}$ had been added to the dispersion at an ionic strength of $119 \mathrm{mmol} / \mathrm{L}$. Fig. 3c shows a data set from an experiment where $\mathrm{Na}_{2} \mathrm{SO}_{4}$ was added to the dispersion at an ionic strength of $238 \mathrm{mmol} / \mathrm{L}$. In the experiments shown in Fig. 3b and 3c, the water distribution developed an asymmetric shape. Drying proceeded heterogeneously.

In order to describe the influence of added salt onto skin formation, a quantitative indicator of vertical heterogeneity is needed. We use the skewness of the profile $\varphi_{w}(z)$ for that purpose. The analysis comprised the following steps: 
a) Normalization: To correct for the sensitivity decline over the film thickness, profile intensities were normalized by dividing by the profile obtained from an elastomer standard. In a second step, the profiles were divided by the function $A-B z$, where the parameters $A$ and $B$ were chosen such that the first profile of any given experiment had a flat top at $\varphi_{\mathrm{w}}(z) \equiv 1-\Phi_{0}$. This step served to normalize the profiles and to remove a slight residual slope in the profiles, which was still present after the first normalization step. The second step assumes a uniform distribution of water at the start of the experiment.

b) Smoothing: An adjacent average according to $\varphi_{i} \rightarrow 1 / 4\left(\varphi_{i-1}+2 \varphi_{i}+\varphi_{i+1}\right)$ was applied. This step removed a systematic even-odd difference between adjacent data points. This artifact is related to insufficiently accurate pulse sequences of the NMR spectrometer.

c) Calculation of the integral amount of free water: The amount of free water is the integral over $\varphi_{\mathrm{w}}(z)$. For the purpose of integration, we have cut all profiles to $\varphi_{w}(z) \equiv 0$ when $\varphi_{w}(z)$ fell to below the noise level. Otherwise, the calculations of higher moments (see below) would have been strongly affected by the noise. The noise level was found to increase with increasing distance from the substrate. Therefore, we used the function $\varphi_{\text {noise }}=\alpha z^{2}$ with $\alpha \sim 6 \times 10^{-6} \mu \mathrm{m}^{-2}$ as the "noise level." d) Calculation of the skewness: The skewness is a model-free indicator of asymmetry. It is defined as

$$
S=\frac{m_{3}^{*}}{\left(m_{2}^{*}\right)^{3 / 2}}
$$

with $m_{3}{ }^{*}=m_{3} / m_{0}$ and $m_{2}{ }^{*}=m_{2} / m_{0}$ being the normalized third and second moments of the distribution, respectively. The $n$-th moment of any given discrete distribution $\varphi_{\mathrm{i}}\left(z_{\mathrm{i}}\right)$ is given by 
$m_{n}=\sum z_{i}^{n} \varphi_{i} \Delta z$

The sum runs over all data points. For the calculation of moments, the origin of the $z$-scale was set such that the first moment vanishes (that is, all $z_{\mathrm{i}}$-s are to be understood as distances to the center of gravity of the profile). Once again, the profiles were cut off when $\varphi_{\mathrm{w}}(z)$ fell below the noise level.

e) Calculation of the solids content: The $z$-averaged solids content, $\langle\Phi(t)\rangle$, was calculated via the relation

$$
\langle\Phi(t)\rangle=1-\frac{m_{0}(t)}{m_{0,0}} \cdot\left(1-\Phi_{0}\right) \cdot \frac{H_{0}}{H(t)}
$$

where $m_{0}(t)$ is the $0^{\text {th }}$ moment of $\varphi_{w}(z)$ at time $t, m_{0,0}$ is the $0^{\text {th }}$ moment at $t=0, \Phi_{0}$ is the solids content at $t=0, H(t)$ is the thickness at time $t$, and $H_{0}$ is the initial thickness. The thickness, $H(t)$, was determined from the MRP profiles. In doing so, we assumed that the upper edge of the water profile coincides with the upper edge of the film. That would not be true if there were a dry, open layer at the top, containing no free water at all. Such a dry surface seems unlikely, especially in the earlier stages of drying. Also, if this were the case, one would expect an extended tail in $\varphi_{w}(z)$ at some intermediate stage in drying (which was not observed). Note that the time derivative of $H(t)$ is not strictly equal to the water evaporation speed, $E$, because the samples were affected by the socalled coffee-stain effect. ${ }^{29}$ That is, some material moved from the center of the film towards the rim (not probed in the MRP experiment), thereby decreasing the measured film thickness in the center for reasons other than water evaporation. 


\section{Diffusing Wave Spectroscopy}

In order to monitor particle mobility in parallel to the water content, a diffusing wave spectroscopy (DWS) setup was installed above the MRP instrument. Briefly, a HeNe laser illuminated the sample from the top. Scattered light was collected from the center of the film by means of an optical fiber equipped with a collimator. The light was fed into a photon correlation spectroscopy (PCS) module. Autocorrelation functions were acquired at a rate of three data traces per minute. The autocorrelation functions were fitted with stretched exponentials of the form

$$
g_{2}\left(\tau^{\prime}\right)-1=A \exp \left(-\left(\frac{\tau^{\prime}}{\tau_{D}}\right)^{\beta}\right)
$$

where $A$ is an amplitude, $\tau_{D}$ is a decay time, and the exponent $\beta<1$ is an indicator of nonexponentiality. Fig. 4 shows a typical autocorrelation curve and a fit. In DWS, $\beta$ cannot be quantitatively translated into dynamical heterogeneity (coexistence of fast and slow dynamics) because the path-length distribution introduces a distribution of decay times even if the medium of interest would yield a single exponential in conventional light scattering. Actually, $\beta$ was found to decrease with drying time in the present work. This matches the expectation of an increasing dynamical heterogeneity as the sample dries from the top to the bottom.

\section{Results and Discussion}

We first discuss crust formation as evidenced by the DWS data. Fig. 5 shows that for a latex containing added salt, the amplitude of the autocorrelation function sometimes sharply drops during an experiment. We refer to the time at which this happen as $T_{\mathrm{S}}$. The corresponding solids content $\left(\Phi_{\mathrm{S}}\right)$ is indicated with a vertical line in Fig. 5. Simultaneous MRP shows that water is present in the 
surface layer, indicating that full coalescence has not created a skin. The drop in amplitude is caused by the (rather sudden) appearance of a static scattering component in the DWS data. A drying latex film is a spatially heterogeneous medium, where solidified and liquid components may coexist. We associate the drop in amplitude with the formation of a solidified layer at the top, which is best described as a crust (Figure 1) until proven otherwise. (As we will discuss later, the surface layer has not coalesced fully to create a skin.) The light scattered from these assemblies of elasticallycoupled particles fluctuates at a time scale longer than one second. The decay time of the autocorrelation function, $\tau_{D}$, goes to infinity (or, more precisely, leaves the experimental window) soon after $T_{S}$. The time stretch during which a crust coexists with a liquid underneath is rather short. Particle diffusion comes to rest a few minutes after the crust has formed.

Interestingly, the crust moves laterally even after Brownian motion has stopped. One can visually observe this movement when imaging the drying film with a camera. The lateral drift of the scatterers causes a rather sizeable variability of the scattering intensity (that is, the count rate as determined by the PCS module) on a time scale of minutes (encircled region in Fig. 5). These fluctuations slow down with continued drying. They almost come to rest at the same time at which the free water has disappeared from the film according to the MRP data. Some limited scattering still persists even after drying is complete because the film surface is rough.

As the comparison between the MRP results and the DWS data shows, there is a strong correlation between the skewness of the water distribution and crust formation (Fig. 5), as detected via DWS. The crust is created when the skewness is at its maximum. The maximum of the skewness occurs when the $z$-averaged particle volume fraction is around 54\%. This volume fraction corresponds to the value where hard-sphere colloids go through the glass transition, $\Phi_{\mathrm{gl} .}{ }^{30}$ A peak of the skewness at this volume fraction is very reasonable. Beyond $\Phi_{\mathrm{gl}}$, diffusional rearrangements 
inside the dense layer at the top are slow. When the density inside the crust has reached $\Phi_{\mathrm{gl}}$, further drying will increase the thickness of the crust, rather than its density. Eventually, the crust will make up the entire film and the skewness falls back to a value close to 0 .

Fig. 6 shows the amplitude of the autocorrelation function, $A$, the decay time $\tau_{D}$, the skewness of the water distribution, and the drying rate $\mathrm{d} H / \mathrm{d} t$ for films containing one of three different salts and for the as-received latex without salt for a reference sample. The ionic strengths of added salts were chosen such that the value of the Debye screening length, $\kappa^{-1},{ }^{23}$ in the dispersion was $0.5 \mathrm{~nm}$, taking the effects of the different valencies (1 or 2) into account. As water evaporates, the salt concentration increases and $\kappa^{-1}$ decreases, accordingly. For the reference sample, $\kappa^{-1}$ is much larger than $0.5 \mathrm{~nm}$ because the electrolyte concentration is naturally lower. The exact value of $\kappa^{-1}$ is not known. Both the surfactant and the initiator are charged, and the degree to which these components are dissolved in the serum is uncertain.

Evidence for a crust layer was found in the DWS data when drying dispersions containing $\mathrm{NaNO}_{3}$ and $\mathrm{NaClO}_{4}$, whereas no such layer was found for the as-received latex and for the sample containing $\mathrm{Na}_{2} \mathrm{SO}_{4}$. The former two samples have a significantly higher skewness than the latter (Fig. 7).

The R-R model in its standard form does not explain why the addition of salt increases the water non-uniformity. In the Stokes-Einstein limit, the diffusivity is not influenced by interparticle forces. Even if the Stokes-Einstein diffusivity is replaced by a cooperative diffusion coefficient (see below), it is not clear, why an ion's position in the Hofmeister series should affect the diffusivity. On the other hand, particle coalescence and the rupture of surfactant membranes, in particular, are among 
the properties following the Hofmeister series. This suggests that membrane rupture induced by added salt aids the development of heterogeneity and possible skin formation.

\section{Estimation of the Peclet number}

In order to more quantitatively describe the role of interparticle potentials in the skin formation process, we estimate the Peclet number for the films investigated in this study, taking interactions into account. Interactions enter the Peclet number via cooperative diffusion. Within the standard picture, crust layers (which can then lead to skin formation) will only develolp when $P e$ 1. $P e$ is defined as the ratio of the time scale at which concentration differences diffusively decay, $\tau_{\text {diff, }}$, to the characteristic drying time, $\tau_{\mathrm{dry}}$, such that:

$$
P e=\frac{\tau_{\text {diff }}}{\tau_{d r y}}=\frac{H_{0}^{2} / D_{\text {coop }}}{H_{0} / E}=\frac{E H_{0}}{D_{\text {coop }}}
$$

In our work, $E$ is obtained from $(\mathrm{d} H / \mathrm{d} t)$. In a departure from previous work, we propose the use of $D_{\text {coop }}$ rather than $D_{0}$ in the expression for $P e$. In the dilute limit, the cooperative diffusion coefficient (governing concentration gradients) is equal to the self-diffusion coefficient (governing Brownian motion and the mean-square displacement of individual particles). For particles of radius $a$ in a continuous medium with a viscosity of $\mu$, both are given by the Stokes-Einstein formula $D_{0}=k_{B} T /(6 \pi \mu a)$, with $T$ the absolute temperature and $k_{\mathrm{B}}$ the Boltzmann constant. At higher concentrations, hydrodynamic interactions as well as static interparticle forces come into play. Usually, these slow down the motion of individual particles (for instance by caging). ${ }^{31}$ At the same time, they speed up the equilibration of concentration distributions via cooperative motion. More quantitatively, we have ${ }^{32}$ 
$D_{\text {coop }}=D_{0} K(\Phi) \frac{4 \pi a^{3}}{3} \frac{1}{k_{B} T}\left(\frac{\mathrm{d} \Pi(\Phi)}{\mathrm{d} \Phi}\right)$

Eq. 6

$K(\Phi)$ is the sedimentation coefficient, accounting for the fact the hydrodynamic backflow is slowed down by the neighboring particles, and $\Pi(\Phi)$ is the osmotic pressure. The sedimentation coefficient can be approximated as ${ }^{33}$

$K(\Phi)=\frac{1-\frac{3}{2} \Phi^{1 / 3}+\frac{3}{2} \Phi^{5 / 3}-\Phi^{2}}{1+\frac{2}{3} \Phi^{5 / 3}}$

Eq. 7 is the analytical solution of a cell model. A spherical particle was assumed to be contained in a second spherical volume with frictionless walls. $\Phi$ is the ratio of the two volumes. For the purpose of this estimate, we identify the parameter $\Phi$ with the solids content. In the range of solids contents of interest here $(\Phi=0.4-0.65), K(\Phi)$ takes values between 0.01 and 0.04 . Eq. 7 shows that $K(\Phi)$ decreases with increasing solids content.

The osmotic pressure, $\Pi(\Phi)$, has three contributions, which originate from electrostatic repulsion, van der Waals attraction, and entropy. We therefore have the three corresponding terms in the expression for $\Pi:^{32,34}$

$\Pi(D) \approx \frac{\sqrt{6}}{A_{h}}\left(F_{E S}(D)+F_{A}(D)\right)+p_{\text {entr }}$ Eq. 8

where $A_{h}$ is the surface of interaction. We approximate $A_{h}$ as the surface of a sphere with radius $a+D / 2$, that is $A_{h} \sim 4 \pi(a+D / 2)^{2} . \quad D$ is the mean distance between particles surfaces, and it is related to $\Phi$ via the relations 
$\frac{\Phi}{\Phi_{R C P}} \approx \frac{a^{3}}{(a+D / 2)^{3}} \approx \frac{a^{3}}{a^{3}+3 D a^{2}}$

$D \approx \frac{a}{3}\left(\frac{\Phi_{R C P}}{\Phi}-1\right)$

$\Phi_{R C P} \approx 63 \mathrm{vol} . \%$ is the solids content at random close-packing. We assume that the particles are hard enough to remain spherical at this solids content. Softer spherical particles would deform and coalesce upon first contact. The parameter $F_{E S}$ in Eq. 8 is the electrostatic force between two charged spheres. For the purpose of this estimate we assume a 1:1 electrolyte and use the weak overlap approximation, ${ }^{23}$ leading to

$$
\begin{aligned}
F_{E S} & \approx \frac{\pi a 64 k_{B} T \rho_{\infty}}{\kappa}\left(\tanh \left(\frac{e \Psi_{0}}{k_{B} T}\right)\right)^{2} \exp (-\kappa D) \\
& =\pi a 0.0482[\text { electrolyte] }]^{1 / 2}\left(\tanh \left(\frac{\Psi_{0}[m V]}{103}\right)\right)^{2}
\end{aligned}
$$

Here $\Psi_{0}$ is the electrical potential at the particle surface and $\rho_{\infty}$ is the electrolyte concentration far away from charged surfaces. A typical value for particles stabilized with a sulphonate-based surfactant is $\Psi_{0} \sim-80 \mathrm{mV}$. Measurements of the zeta potential on the dispersions used in this study are consistent with this value. As pointed out earlier, $\kappa^{-1}=0.5 \mathrm{~nm}$ in the dispersion at the salt concentrations used. In general, $\kappa$ is given by

$$
\kappa^{2}=\left(\frac{\sum_{i} \rho_{\infty i} e^{2} z_{i}^{2}}{\varepsilon \varepsilon_{0} k_{B} T}\right)
$$


where the index $i$ runs over all ionic species, $z$ is the valency, $\rho_{\infty i}$ is the concentration (in ions per $\mathrm{m}^{3}$ ) far away from charged surfaces, $\varepsilon$ is the relative dielectric permittivity of the liquid, and $\varepsilon_{0}$ is the dielectric permittivity of vacuum. Note that $\rho_{\infty i}$ increases when water evaporates so that $\kappa^{-1}$ decreases during drying. This effect was taken into account in the calculations presented later. However, whereas in the study by Erkselius et al $^{12}$ the particles became unstable because of the rise in ionic concentration during drying, our calculations indicate that CCC is not reached in our system until after the time at which particle close-packing occurs.

The van der Waals force between spherical particles is given by ${ }^{35}$

$$
\begin{aligned}
& F_{A}=-\frac{\mathrm{d} V_{A}}{\mathrm{~d} D} \\
& V_{A} \approx-\frac{A_{H}}{6 D}\left[\frac{2 a^{2}}{D^{2}+4 a D}+\frac{2 a^{2}}{(D+2 a)^{2}}+\ln \left(1-\frac{4 a^{2}}{(D+2 a)^{2}}\right)\right]
\end{aligned}
$$

$A_{H}$ is the Hamaker constant. Using $A_{H} \sim\left(A_{p o l}{ }^{1 / 2}-A_{w}{ }^{1 / 2}\right)^{2}$ and further using $A_{p o l} \sim 8.2 \times 10^{-20} \mathrm{~J}$ and $A_{w} \sim 3.7 \times 10^{-20} \mathrm{~J},{ }^{36}$ we arrive at $A_{H} \sim 0.9 \times 10^{-20} \mathrm{~J}$. Finally, the entropic pressure can be approximated $\mathrm{as}^{37}$

$$
p_{\text {entr }} \approx \frac{k_{B} T}{4 \pi a^{3} / 3} \Phi\left(\frac{1+\Phi+\Phi^{2}-0.67825 \Phi^{3}-\Phi^{4}-0.5 \Phi^{5}-6.028 \exp (\xi(7.9-3.9 \xi)) \Phi^{6}}{1-3 \Phi+3 \Phi^{2}-1.04305 \Phi^{3}}\right)
$$

The term in brackets is the compression factor of a hard-sphere fluid. All spheres are assumed to be of the same size. $\xi=\sqrt{2} \pi / 6-\Phi$ is the difference between the particle volume fraction and the maximum particle volume fraction $(\approx 0.74)$. The entropic pressure is small compared to the 
electrostatic pressure unless one considers a solids content very close to close packing. At $\Phi \approx 0.74$, $p_{\text {entr }}$ sharply diverges. This range of solids contents is not considered here because coalescence will modify the picture.

Using equations 6 to 12, one can estimate $D_{\text {coop }}$ and its dependence on solids content and added salt. The result of this estimate is shown in Fig. 8. The particle volume fraction, $\Phi$, has been limited to $\Phi<60 \%$ because the model does not account for coalescence. Coalescence must enter the picture at around the point of particle close-packing. The input parameters to the calculation are the particle radius, $a$ (determined by DLS as $90 \mathrm{~nm})$, the surface potential $\Psi_{0},(-80 \mathrm{mV})$, and the Hamaker constant, $A_{H}$ (as chosen above). Note that this model does not differentiate between different types of ions with the same valency. The only input parameters into the DLVO theory are the surface potential, the ions' valencies, the ionic strength, and the particles' Hamaker constant.

Consider first the case of a Debye length larger than $2 \mathrm{~nm}$. This would correspond to the reference experiments conducted without added salt. For these cases, the cooperative diffusion coefficient is much larger than the Stokes-Einstein value and weakly depends on $\Phi$. This is a consequence of the fact that electrostatic repulsion softens the interparticle potential. $D_{\text {coop }}$ may decrease at close-packing due to the van der Waals attractions. The value of $D_{\text {coop }}$ is more than 100 times greater than $D_{0}$. Inserting the Stokes-Einstein value $D_{0}=k_{\mathrm{B}} T /(6 \pi \mu a)$ and using a viscosity of $\mu=1 \mathrm{mPa}$ s (corresponding to water), $D_{\text {coop }}$ comes out to be around $10^{-5} \mathrm{~cm}^{2} / \mathrm{s}$. Inserting this value into Eq. 5 and further using $H_{0} \sim 610^{-2} \mathrm{~cm}$ as well as a drying time of $\tau_{d r y} \sim 8100 \mathrm{~s}$, we arrive at the following estimate for $P e$ : 
$P e=\frac{\tau_{d i f f}}{\tau_{d r y}} \approx \frac{E H_{0}}{D_{\text {coop }}} \approx \frac{H_{0}^{2}}{T_{\text {dry }} D_{\text {coop }}} \approx \frac{\left(6 \times 10^{-2} \mathrm{~cm}\right)^{2}}{\left(10^{-5} \mathrm{~cm}^{2} / \mathrm{s}\right) \times 8100 \mathrm{~s}} \approx 0.04$

For $P e<1$, particle accumulation at the film surface is not expected according to the R-R argument. Particle accumulation is a prerequisite for crust formation. Indeed, a crust is not observed in our experiments without added salt, which is consistent with $P e \approx 0.04$.

At a Debye length of $0.5 \mathrm{~nm}$, the situation is changed. At $\Phi \sim 0.5, D_{\text {coop }}$ is about equal to the Stokes-Einstein value, $D_{0}$. Inserting this value into Eq. 14 , we find $P e \sim 4$, which would predict non-uniform drying and particle accumulation at the film surface, as was observed experimentally with the addition of salt. A lowered value of $D_{\text {coop }}$ at the same time implies a faster motion on the local scale. Because the interparticle repulsion has been reduced relative to the experiments without added salt, the particles feel the caging by their neighbors to a lesser extent. The short-time selfdiffusion coefficient therefore increases. DWS is mostly sensitive to the variability of next-neighbor distances. These fluctuate faster if the repulsion is reduced. The autocorrelation time in the DWS data, $\tau_{D}$, therefore decreases.

However, even in the presence of added salt, $D_{\text {coop }}$ sharply increases as the particle volume fraction, $\Phi$, approaches 0.6 . The added salt screens the electrostatic interactions at distances larger than the Debye length, but at short distances, the electrostatic interaction is still strong. Observe that Eq. 10 contains the square root of the electrolyte concentration in the numerator. When the particles approach closely, the osmotic pressure of the counter ions in the gap becomes very large.

The consequences for the drying process are the following. At moderate concentrations $(\Phi \sim 0.5), P e$ is larger than one and particles therefore accumulate at the top. The snow-plough effect is active. However, the Peclet number inside the densified layer sharply increases and 
particles will still escape skin formation because they are driven away from the film-air interface, once the local solids content approaches 0.6. Moreover, if particles are sufficiently stable against coalescence, skin formation will not occur under these conditions. However, at $\Phi \sim 0.6$, the particles have approached closely and coalescence is imminent. Importantly, the data indicate that the rate of coalescence depends on the type of ion employed. Coalescence is among the phenomena affected by an ion's position in the Hofmeister series.

Addition of ions affects the skewness in two ways. Firstly, it will (always) induce vertical heterogeneity because it will decrease $D_{\text {coop. }}$. Secondly, the ions may encourage coalescence (and thereby further increase the skewness), if the respective ions are destabilizing. We checked for the destabilizing action of $\mathrm{Na}_{2} \mathrm{SO}_{4}, \mathrm{NaNO}_{3}$, and $\mathrm{NaClO}_{4}$ by determining the critical coagulation concentrations (CCC), which were 2.7, 0.96, and $0.94 \mathrm{~mol} / \mathrm{L}$ for $\mathrm{Na}_{2} \mathrm{SO}_{4}, \mathrm{NaNO}_{3}$, and $\mathrm{NaClO}_{4}$, respectively. The CCC is lowest for $\mathrm{NaClO}_{4}$, which also induces the largest skewness in drying.

It is interesting to compare the estimates of $D_{\text {coop }}$ derived using eqs. $6-12$ to experimental values derived from the profiles, $\varphi_{w}(z)$, and Fick's first law $\left(J=-D_{\text {coop }} \nabla c\right)$. Note that for binary, incompressible systems, $D_{\text {coop }}$ is always the same for both components, and we can obtain $D_{\text {coop }}$ from the evolution of $\varphi_{\mathrm{w}}(z)$. Both the flow rate of water towards the surface, $J$, and the gradient of water concentration, $\nabla c$, can be extracted from experiments. The calculation of the flow rate from $\varphi_{\mathrm{w}}(z, t)$ is provided in appendix A. One has

$\frac{J_{w}}{\rho_{w}}=-\frac{1}{\left(1-\bar{\varphi}_{w}\right)} \frac{\mathrm{d} m_{0, w}}{\mathrm{~d} t}+\left(\frac{\bar{\varphi}_{w}}{\left(1-\bar{\varphi}_{w}\right)}-\varphi_{w}(H)\right) \frac{\mathrm{d} H}{\mathrm{~d} t}$

where $\rho_{w}$ is the density of water and $\bar{\varphi}_{w}$ is the z-averaged water content. 
First, consider the experiment shown in Fig. 3a, which did not show crust or skin formation. At a drying time of $t=100 \mathrm{~min}$, the analysis yields $\bar{\varphi}_{w} \sim 40 \%, \varphi_{w}(H) \sim 37 \%, \mathrm{~d} m_{0, w} / \mathrm{d} t \sim-3.8 \mu \mathrm{m} / \mathrm{min}$, $\mathrm{d} H / \mathrm{d} t \sim-2.9 \mu \mathrm{m} / \mathrm{min}, \quad \mathrm{d} c / \mathrm{d} z \sim 5.210^{8} \mathrm{~g} / \mathrm{m}^{4}, \quad$ and $\quad D_{\text {coop }} \sim 1.710^{-6} \mathrm{~cm}^{2} / \mathrm{s} \quad$ (Fig. 9a). Using $\tau_{d r y} \sim 8100 \mathrm{~s}$ and $H_{0} \sim 5.510^{-2} \mathrm{~cm}$, we estimate the Peclet number as $P e \sim 0.2$ (Eq.14). Crust formation is therefore not predicted. As a second example, consider the experiment shown in Fig. 3b, which did show crust formation. At a drying time of $t=102 \mathrm{~min}$, we have $\bar{\varphi}_{w} \sim 46 \%$, $\varphi_{w}(H) \sim 34 \%, \quad \mathrm{~d} m_{0, w} / \mathrm{d} t \sim-3.6 \mu \mathrm{m} / \mathrm{min}, \quad \mathrm{d} H / \mathrm{d} t \sim-2.8 \mu \mathrm{m} / \mathrm{min}, \quad \mathrm{d} c / \mathrm{d} z \sim 1.9 \quad 10^{9} \mathrm{~g} / \mathrm{m}^{4}, \quad$ and $D_{\text {coop }} \sim 4.610^{-7} \mathrm{~cm}^{2} / \mathrm{s}$ (Fig. 9b). Using $\tau_{d r y} \sim 8520 \mathrm{~s}$ and $H_{0} \sim 5.210^{-2} \mathrm{~cm}$, we estimate the Peclet number as $P e \sim 0.7$. Although crust formation is not predicted by this $P e$, the expected increase in $P e$ is observed compared to the first example. The same kind of analysis leads to $P e \sim 0.7$ for the experiment shown in Fig. 3c. Clearly, the addition of salt did slow down cooperative diffusion, but the effect amounts to a factor of less than 4, while Eq. 6-12 predicted a factor of around 100 . Presumably, the discrepancy can be attributed to elastic contacts between particles touching each other. Once the particles have partly coalesced, these contacts again increase the cooperativity of particle movement. We further elaborate on the dynamics of this elastic network in the following section.

\section{Estimation of particle viscosity and particle deformation rate}

In order to model the evolution of $\varphi_{w}(z)$ at concentrations beyond the volume fraction of the glass transition of $\Phi_{\mathrm{gl}} \sim 53 \mathrm{vol} \%$, one would need to include the elastic forces inside the network of (randomly-packed) contacting spheres. Particles will pack in a crust at the top of the film during drying without actually forming a skin. Two cases have to be distinguished. The particles may remain entirely separate. This case would, for instance, happen with high- $T_{g}$ materials or with those 
that are very well stabilized against aggregation. A second case is partial coalescence. This would typically happen with harder particles (at a temperature just above the $T_{\mathrm{g}}$ ) which are not well stabilized against aggregation. When such particles touch, the surfactant membranes break and the sintering process is initiated. However, the sintering is so slow that the water channels do not actually close before drying is complete. Such a layer might be called an "open skin" (Figure 1). Below, we argue that the films investigated here develop such an open skin.

We do not attempt a description of the transition regime, where there is a loosely-coupled elastic network still experiencing electrostatic repulsion. However, once the elastic network has become so dense that particle deformation dominates the film formation kinetics, the R-R model again applies. Particle deformation limits the speed of film formation if the particles are sufficiently hard. Since the $T_{g}$ of the films formed here is around $18{ }^{\circ} \mathrm{C}$, particle deformation is expected to be slow. In passing, we note that a model based on foam drainage applies in the opposite limit of soft spheres. ${ }^{38}$

The R-R model uses the terminology of viscoelasticity and we follow that usage. Note, however, that one might, in principle, also add a term describing hydrostatic pressure exerted by the coalescence into Eq. 8, thereby staying within the conceptual frame of eqs. 6-12. For densely packed spheres, the R-R model relates the rate of drying to the viscosity of the particles. Skin formation only occurs if the particles are sufficiently soft. Otherwise, the particles do not form a dense layer. As already noted, particle deformability is described by a dimensionless parameter $\bar{\lambda}=\eta_{0} a E /\left(\gamma_{\mathrm{wat}} H_{0}\right)$. As we show in appendix $\mathrm{B}, \bar{\lambda}$ can be deduced from $\varphi_{\mathrm{w}}(z, t)$ via

$$
\bar{\lambda}=\frac{7}{5} \frac{\bar{\gamma}\langle\Phi\rangle^{2}}{\Phi_{R C P} \tau_{d r y}}\left(\frac{\partial \Phi}{\partial t}\right)^{-1}
$$


The parameter $\bar{\gamma}$ is ratio of the particle/water interfacial tension and the air/water interfacial tension, which was assumed to be 0.5 in this estimate. The volume fraction at close-packing, $\Phi_{\mathrm{RCP}}$, is taken to be 63 vol.\%. Using the profiles shown in Fig. 3 and analyzing the time stretch between $t=80 \mathrm{~min}$ and $t=105 \mathrm{~min}$, we find $\bar{\lambda} \sim 0.44, \bar{\lambda} \sim 0.40$, and $\bar{\lambda} \sim 0.44$ for the pure dispersion and for the cases of added $\mathrm{MgCl}_{2}$ and added $\mathrm{Na}_{2} \mathrm{SO}_{4}$, respectively.

Given that $\bar{\lambda}$ is close to one, we conclude that the layer formed at the top is not a closed film (a "skin" in the narrow sense of the word). The same conclusion is reached from Fig. 5e and 6d. Whereas there is clear evidence of a static scatterer at volume fractions above $\Phi_{\mathrm{S}}$, the rate of evaporation $\mathrm{d} H / \mathrm{d} t$ does not slow down after the elastic layer has formed. Water channels remain open, because the particle deformation rate is small. Moreover, noting that the experimental temperature of $c a .25{ }^{\circ} \mathrm{C}$ is within approximately $7{ }^{\circ} \mathrm{C}$ of the polymer's $T_{\mathrm{g}}$, the R-R process model ${ }^{8}$ does not predict wet sintering, thus preventing skin formation. An "open skin" (see Figure 1) is expected and is consistent with experimental observations..

\section{Conclusions}

Applying magnetic resonance profiling and photon correlation spectroscopy simultaneously in the same experiment, we have investigated the vertical heterogeneity of the water distribution and possible skin formation in drying polymer dispersions. Using the two techniques together provides a complete picture of the surface layer with information both on the particle dynamics (coupling) and the composition. We propose to use the skewness of the profile $\varphi_{w}(z)$ as an indicator of heterogeneity. The skewness peaks at a $z$-averaged solids content of around $53 \mathrm{vol} \%$, which is close to the volume fraction where hard-sphere colloids go through the glass transition. At the same solids content, one sometimes observes a strong static component in the scattering, which originates from a 
crust layer. Addition of salt increases the skewness and the likelihood of subsequent skin formation. The strength of the effect depends on the particular type of ion. These results suggest that the destabilizing effect of the ions onto the surfactant membranes separating the particles promote heterogeneous drying. The order in which the ions influence destabilization is suggestive of a Hofmeister effect. In turn, both the speed of diffusion and the rate of coalescence have an influence on skin formation. Particle accumulation during drying was successfully predicted by a Peclet number that considered the cooperative particle diffusion, rather than the standard Stokes-Einstein diffusivity.

\section{Acknowledgements}

This work was partly funded by the European Commission under contract IP 011844-2 (NAPOLEON). We gratefully acknowledge helpful discussions with Peter McDonald (University of Surrey) and Ola Karlsson (Lund University), as well as miniemulsion polymerization by Raquel Rodriguez and Maria Barandarian (University of the Basque Country, San Sebastian).

\section{Appendix A: Calculation of the flow rate from $\varphi_{w}(z, t)$}

For the experiments reported here, the calculation of the flow rate, $J$, entails a complication because these samples did experience lateral flow (i.e. the coffee-stain effect). Material was moving laterally. The time derivative of the amount of water in the observation volume (the $0^{\text {th }}$ moment of $\left.\varphi_{w}(z)\right)$ has two contributions, which are evaporation and laterally diverging flow due to the coffeestain effect. We have

$$
\frac{\mathrm{d} m_{0, w}}{\mathrm{~d} t}=-E-\bar{\varphi}_{w} H \vec{\nabla}_{\perp} \cdot \vec{v}_{\perp}
$$


where $E$ is the evaporation rate, $\bar{\varphi}_{w}$ is the $z$-averaged water content, $\vec{v}$ is the z-averaged lateral speed of motion and the index $\perp$ denotes in-plane directions $(x$ and $y)$. The decrease of the total amount of polymer has only one source, which is the diverging flow. We have

$\frac{\mathrm{d}}{\mathrm{d} t} m_{0, p o l}=\frac{\mathrm{d}}{\mathrm{d} t}\left(H-m_{0, w}\right)=-\bar{\varphi}_{p o l} H \vec{\nabla}_{\perp} \cdot \vec{v}_{\perp}=-\left(1-\bar{\varphi}_{w}\right) H \vec{\nabla}_{\perp} \cdot \vec{v}_{\perp}$

Eq. 18

In the two equations above, we have assumed the speed $\vec{v}$ is about the same for water and the polymer. From Eq. 17 and Eq. 18, we obtain the evaporation rate as

$$
\begin{aligned}
E & =-\frac{\mathrm{d} m_{0, w}}{\mathrm{~d} t}-\bar{\varphi}_{w} H \vec{\nabla}_{\perp} \cdot \vec{v}_{\perp} \\
& =-\frac{\mathrm{d} m_{0, w}}{\mathrm{~d} t}+\frac{\bar{\varphi}_{w}}{\left(1-\bar{\varphi}_{w}\right)} \frac{\mathrm{d}}{\mathrm{d} t}\left(H-m_{0, w}\right) \\
& =-\frac{1}{\left(1-\bar{\varphi}_{w}\right)} \frac{\mathrm{d} m_{0, w}}{\mathrm{~d} t}+\frac{\bar{\varphi}_{w}}{\left(1-\bar{\varphi}_{w}\right)} \frac{\mathrm{d} H}{\mathrm{~d} t}
\end{aligned}
$$

Eq. 19

In the absence of the coffee-stain effect, $\mathrm{d} H / \mathrm{d} t$ is equal to $\mathrm{d} m_{0, w} / \mathrm{d} t$ and the evaporation rate is the same as the time derivative of the $0^{\text {th }}$ moment. The flow rate close to the top of the film is given by

$$
\frac{J_{w}}{\rho_{w}} \approx E-\varphi_{w}(H) \frac{\mathrm{d} H}{\mathrm{~d} t}
$$

where $\rho_{w}$ is the density of water. Inserting Eq. 19 into Eq. 20 yields

$$
\frac{J_{w}}{\rho_{w}}=-\frac{1}{\left(1-\bar{\varphi}_{w}\right)} \frac{\mathrm{d} m_{0, w}}{\mathrm{~d} t}+\left(\frac{\bar{\varphi}_{w}}{\left(1-\bar{\varphi}_{w}\right)}-\varphi_{w}(H)\right) \frac{\mathrm{d} H}{\mathrm{~d} t}
$$




\section{Appendix B: Relation between $\bar{\lambda}$ and drying rate for strongly coupled elastic networks}

We start out from Eq. 85 in Ref. 8, which states that

$\Phi=\frac{\Phi_{r c p}}{1-\varepsilon}$

Eq. 22

$\varepsilon$ is a volume-averaged strain. Rearranging and taking the time derivative yields

$$
\frac{\partial \varepsilon}{\partial t}=\frac{\Phi_{r c p}}{\Phi^{2}} \frac{\Phi}{\partial t}
$$

At times were the spheres densely packed, the water distribution again is rather homogeneous and one can use $\Phi \sim<\Phi>$. Eq. 84 in Ref. 8 (note the erratum ${ }^{39}$ ) relates the strain rate, $\partial \varepsilon / \partial t$, to $\bar{\lambda}$ via

$$
\frac{\partial \varepsilon}{\partial \bar{t}}=\frac{7}{5} \frac{\bar{\gamma}}{\bar{\lambda}}
$$

where $\bar{t}=t E / H$ is a rescaled time. We approximate $E / H$ by the inverse total time of drying, $\tau_{\text {dry }}$ (cf. Eq. 14). The parameter $\bar{\gamma}$ is ratio of the particle/water interface tension to the air/water interface tension. Inserting and rearranging yields (cf. Eq. 16)

$\bar{\lambda}=\frac{7}{5} \frac{\bar{\gamma}\langle\Phi\rangle^{2}}{\Phi_{R C P} \tau_{d r y}}\left(\frac{\partial \Phi}{\partial t}\right)^{-1}$ 
Published in Langmuir (2008) 24(14):7580-7589

\section{References}

1 Keddie, J. L. Materials Science \& Engineering R-Reports 1997, 21, 101-170.

2 Steward, P. A.; Hearn, J.; Wilkinson, M. C. Advances in Colloid and Interface Science 2000, 86, 195-267.

3 Winnik, M. A. Current Opinion in Colloid \& Interface Science 1997, 2, 192-199.

4 Routh, A. F.; Russel, W. B. Aiche Journal 1998, 44, 2088-2098.

5 Salamanca, J. M.; Ciampi, E.; Faux, D. A.; Glover, P. M.; McDonald, P. J.; Routh, A. F.; Peters, A.; Satguru, R.; Keddie, J. L. Langmuir 2001, 17, 3202-3207.

6 Routh, A. F.; Russel, W. B. Industrial \& Engineering Chemistry Research 2001, 40, 4302-4308.

7 Gorce, J. P.; Bovey, D.; McDonald, P. J.; Palasz, P.; Taylor, D.; Keddie, J. L. European Physical Journal E 2002, 8, 421-429.

8 Routh, A. F.; Russel, W. B. Langmuir 1999, 15, 7762-7773.

9 Sheetz, D. P. Journal of Applied Polymer Science 1965, 9, 3759.

10 Mallégol, J.; Bennett, G.; Dupont, O.; McDonald, P.J.; Keddie, J.L. Journal of Adhesion 2006, 82, 217.

11 Van der Wel G.K.; Adan O.C.G. Progress in Organic Coatings 1999, 37, 1.

12 Erkselius S.; Wadsö L.; Karlsson O.J. Journal of Colloid \& Interface Science, 2008, 317, 83.

13 Yang Y.-K.; Li H.; Wang F. Journal of Adhesion Science and Technology, 2003, 17, 1741.

14 Brochard-Wyart, F.; de Gennes, P. G. Science 2003, 300, 441-441.

15 Routh A.F.; Zimmerman W.B. Chemical Engineering Science 2004, 59, 2961.

16 Ma, Y.; Davis, H. T.; Scriven, L. E. Progress in Organic Coatings 2005, 52, 46-62.

17 Ekanayake P.; McDonald P.J.; Keddie J.L. to appear in European Physical Journal Special Topics.

18 Glover, P. M.; Aptaker, P. S.; Bowler, J. R.; Ciampi, E.; McDonald, P. J. Journal of Magnetic Resonance 1999, 139, 90-97.

19 McDonald, P. J.; Newling, B. Reports on Progress in Physics 1998, 61, 1441-1493.

20 Weitz, D. A.; Pine, D. In Dynamic Light Scattering; Brown, W., Ed.; Oxford University Press: 1992.

21 Schmidt M.; Krieger S.; Johannsmann D. Progress in Colloid \& Polymer Science, 1997, 104, 191. 
Published in Langmuir (2008) 24(14):7580-7589

22 Narita, T.; Beauvais, C.; Hebraud, P.; Lequeux, F. European Physical Journal E 2004, 14, 287-292.

23 Israelachvili, J. N. Surface and Intermolecular Forces; Academic Press, 1991.

24 Hofmeister F.; Naunyn-Schmiedebergs F. Archives of Pathology and Laboratory Medicine, 1888, 24, 247.

25 Kunz, W.; Lo Nostro, P.; Ninham, B. W. Current Opinion in Colloid \& Interface Science 2004, 9, 1-18.

26 Batchelor J.D.; Olteanu A.; Tripathy A.; Pielak G.J. Journal of the Amercian Chemical Society, 2004, 126, 1958.

27 Gurau M.C.; Lim S.-M.; Castellana E.T.; Albertorio F.; Kataoka S.; Cremer P.S. Journal of the Amercian Chemical Society, 2004, 126, 10522.

28 Boström M.; Williams D.R.M.; Ninham B.W. Physical Review Letters 2001, 87, 168103.

29 Deegan, R. D.; Bakajin, O.; Dupont, T. F.; Huber, G.; Nagel, S. R.; Witten, T. A. Nature 1997, 389, 827829.

30 Pusey, P. N.; Vanmegen, W. Nature 1986, 320, 340-342.

31 McPhie, M. G.; Nagele, G. Journal of Chemical Physics 2007, 127, 034906.

32 Bowen, W. R.; Mongruel, A. Colloids and Surfaces a-Physicochemical and Engineering Aspects 1998, $138,161-172$.

33 Happel, J.; Brenner, H. Low Reynolds Number Hydrodynamics; Kluwer, 1973.

34 Bowen, W. R.; Jenner, F. Chemical Engineering Science 1995, 50, 1707-1736.

35 Bowen, W. R.; Jenner, F. Advances in Colloid and Interface Science 1995, 56, 201-243.

36 Kaneda, I.; Vincent, B. Journal of Colloid and Interface Science 2004, 274, 49-54.

37 Hall, K. R. Journal of Chemical Physics 1972, 57, 2252.

38 Narita, T.; Hebraud, P.; Lequeux, F. European Physical Journal E 2005, 17, 69-76.

39 Routh, A. F.; Russel, W. B. Langmuir 2001, 17, 7446-7447. 
Published in Langmuir (2008) 24(14):7580-7589

\section{Figure Captions}

Fig. 1: Sketches of various forms of homogeneous or heterogeneous drying. The dark lines denote the surfactant membranes separating the particles.

Fig. 2: Sketch of the experimental setup. The sample is situated in a strongly heterogeneous magnetic field. The NMR spectrometer acquires profiles of the free water $\varphi_{w}(z)$ at a rate of about 1 data trace per 2 minutes. In parallel, the particle mobility is monitored from the top by Photon Correlation Spectroscopy (PCS).

Fig. 3 A series of distributions $\varphi_{\mathrm{w}}(z)$ acquired in the presence of added salt at regular intervals throughout the entire drying time (shown in the third dimension). The NMR intensity on the vertical axis is proportional to $\varphi_{\mathrm{w}}(z)$. Panel (a) shows the reference experiment on the latex applied as received. For this case, the distribution is rather symmetric throughout the entire process of drying. Addition of $\mathrm{MgCl}_{2}$ at $46 \mathrm{mmol} / \mathrm{L}$ and $\mathrm{NaClO}_{4}$ at $238 \mathrm{mmol} / \mathrm{L}$ increases the asymmetry (panels $\mathrm{b}$ and c, respectively). At later times in (b) and (c), there is a step in $\varphi_{w}(z)$, separating the skin and the dilute dispersion below.

Fig. 4: A typical autocorrelation curve of the scattered light. The line is a fit with the function $A \exp \left(-\left(\tau^{\prime} / \tau_{\mathrm{D}}\right)^{\beta}\right)$

Fig. 5: A typical DWS/MRP data set. This particular latex film contained $\mathrm{MgCl}_{2}$ at an ionic strength of $119 \mathrm{mmol} / \mathrm{L}$. a) Count rate of the DWS instrument, which is a measure of turbidity. The turbidity does not decrease to zero because the surface remains rough after drying. The dotted circle shows noise attributed to the lateral drift of a "skin layer." b) Amplitude of the autocorrelation function, $A$. The amplitude sharply drops at the time where a static scatterer (a skin) forms at the film surface. c) Autocorrelation time $\tau_{\mathrm{D}} \cdot \tau_{\mathrm{D}}$ increases with drying time, reflecting the slowing down of Brownian 
motion. d) Skewness of water distributions, $\varphi_{w}(z)$ obtained from MRP. e) Drying rate, $\mathrm{d} H / \mathrm{d} t$. The skewness peaks at about the same at which the skin develops. The vertical line shows the $z$-averaged solids content at which a solidified layer appears at the top of the film, $\Phi_{\mathrm{S}}$. The drying rate remains about constant after the solidified layer appears.

Fig. 6: Comparison of the influence of three different types of salts. The increase in ionic strength due to the addition of salt was $238 \mathrm{mmol} / \mathrm{L}$ in all cases. For $\mathrm{NaNO}_{3}$ and $\mathrm{NaClO}_{4}$, one finds skin formation, while a skin does not form for $\mathrm{Na}_{2} \mathrm{SO}_{4}$ and for the pure dispersion. Correlating well to this finding, the skewness is highest for $\mathrm{NaNO}_{3}$ and $\mathrm{NaClO}_{4}$. In the initial part of the drying, the autocorrelation time slightly decreases upon the addition of salt. This can be explained as a reduction in the local caging. DWS mostly probes motion on the local scale, which is governed by the short-time self-diffusion coefficient. This parameter increases as the electrostatic interaction is reduced.

Fig. 7: Maximum skewness averaged over three experiments for the pure dispersion and dispersions containing $\mathrm{Na}_{2} \mathrm{SO}_{4}, \mathrm{NaNO}_{3}$, and $\mathrm{NaClO}_{4}$ at an ionic strength of $238 \mathrm{mmol} / \mathrm{L}$.

Fig. 8: Cooperative diffusion coefficients calculated according to eqs. 6 to 12 . The input parameters are $\Psi_{0}=-80 \mathrm{mV}, A_{H}=0.910^{-10} \mathrm{~J}$, and $a=90 \mathrm{~nm}$. For $\kappa^{-1}>2 \mathrm{~nm}$ (corresponding to ionic strength of $23 \mathrm{mmol} / \mathrm{L}$ ), the $D_{\text {coop }}$ is about a factor of 100 higher than the Stokes-Einstein value, $D_{0}$.

Fig. 9: An estimate of the cooperative diffusion coefficient can be based on the concentration gradient and the vertical flow rate of water. The profiles correspond to the experiments shown in Fig. 3 ( $t=100 \mathrm{~min}, 102 \mathrm{~min}$ and $102 \mathrm{~min}$ for the top to the bottom panel, respectively). 
Fig. 1

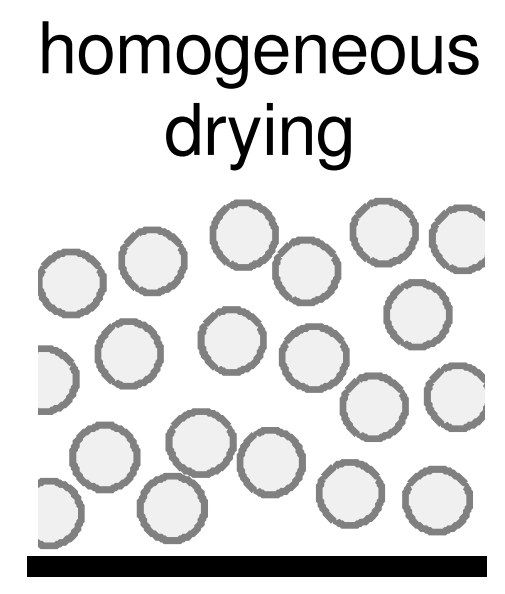

\section{crust}

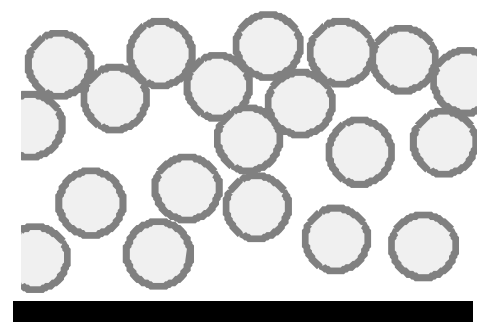

open skin

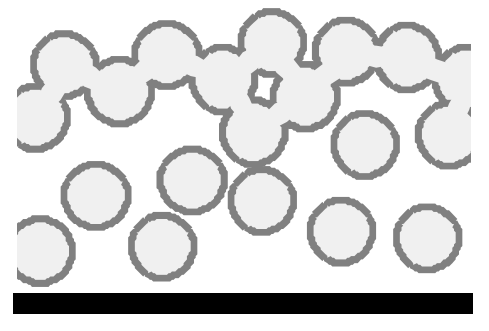

skin

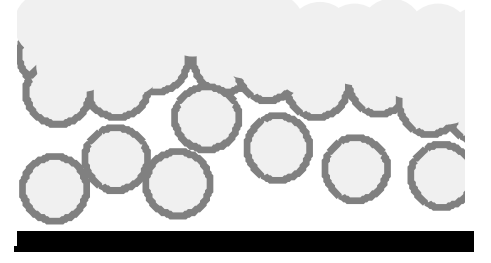


Published in Langmuir (2008) 24(14):7580-7589

Fig. 2

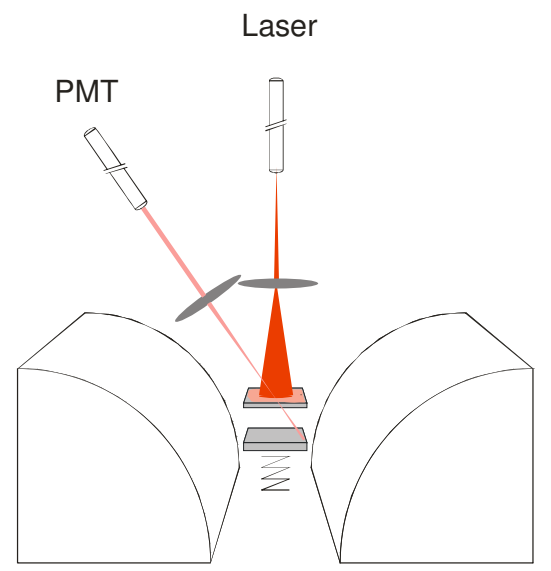

Fig. 3

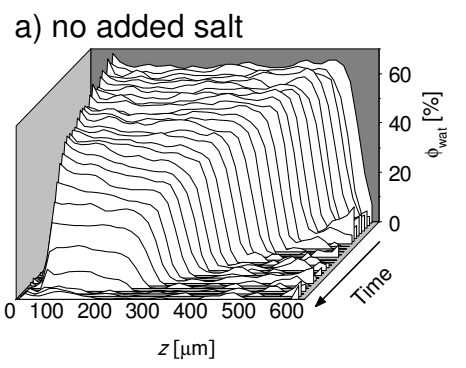

b) $46 \mathrm{mmol} / \mathrm{L}$ added $\mathrm{MgCl}_{2}$

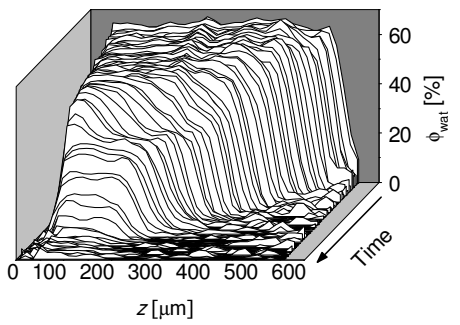

c) $274 \mathrm{mmol} / \mathrm{L}$ added $\mathrm{NaClO}_{4}$

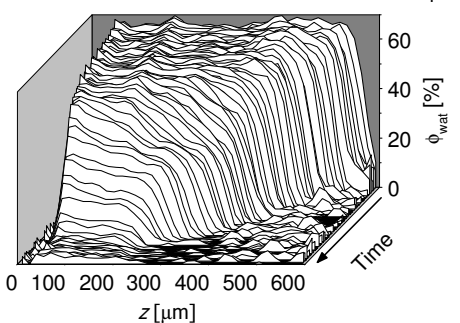


Published in Langmuir (2008) 24(14):7580-7589

Fig. 4

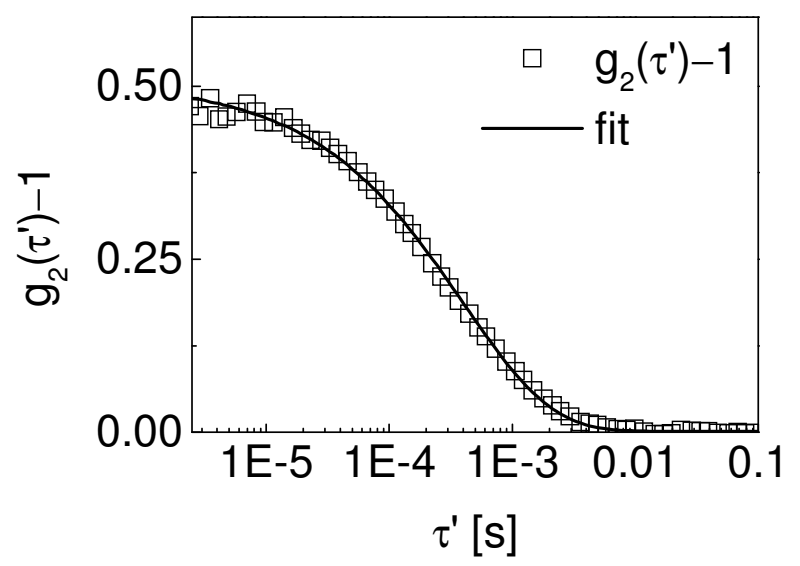

Fig. 5

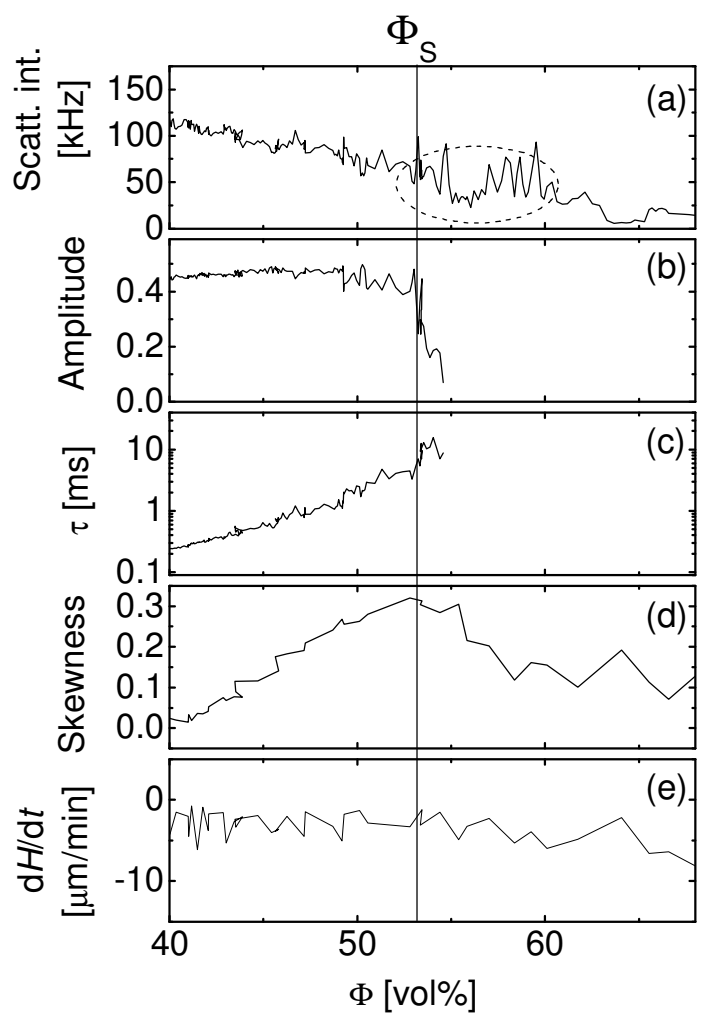


Published in Langmuir (2008) 24(14):7580-7589

Fig. 6

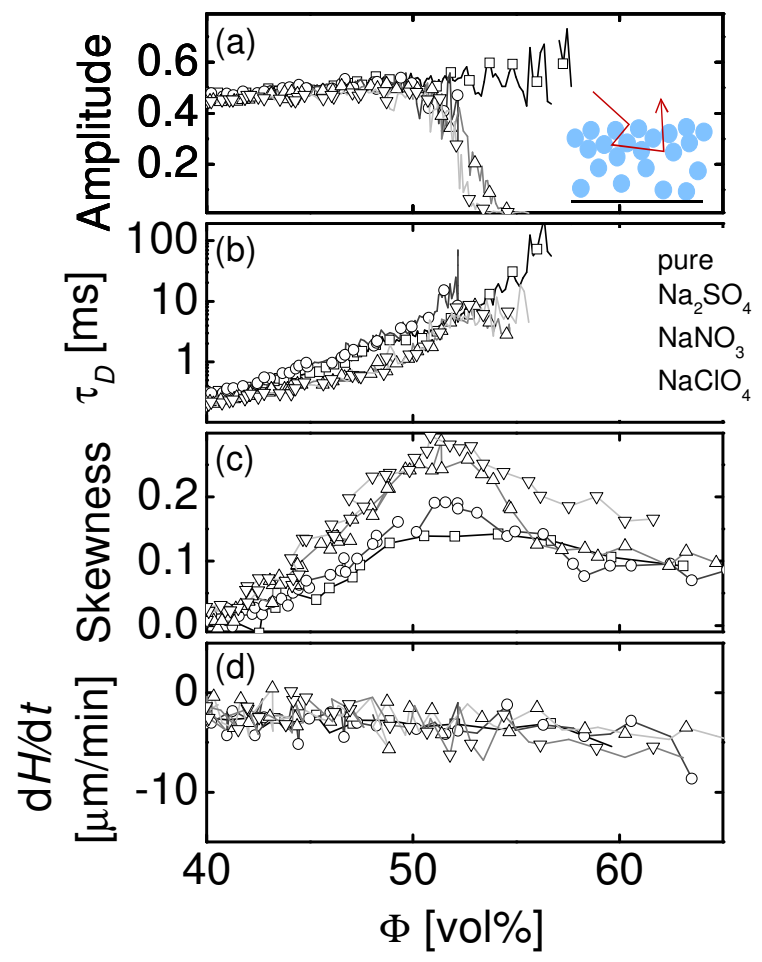

Fig.7

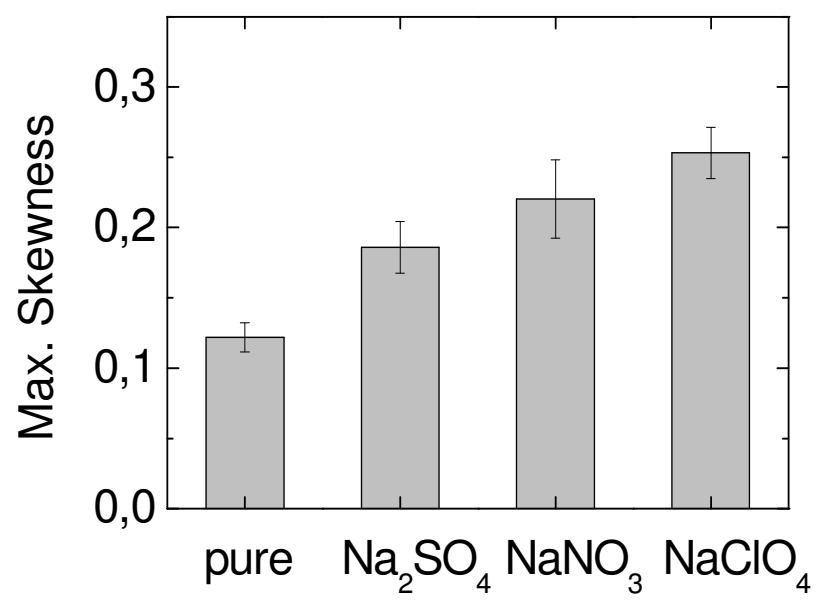


Fig. 8

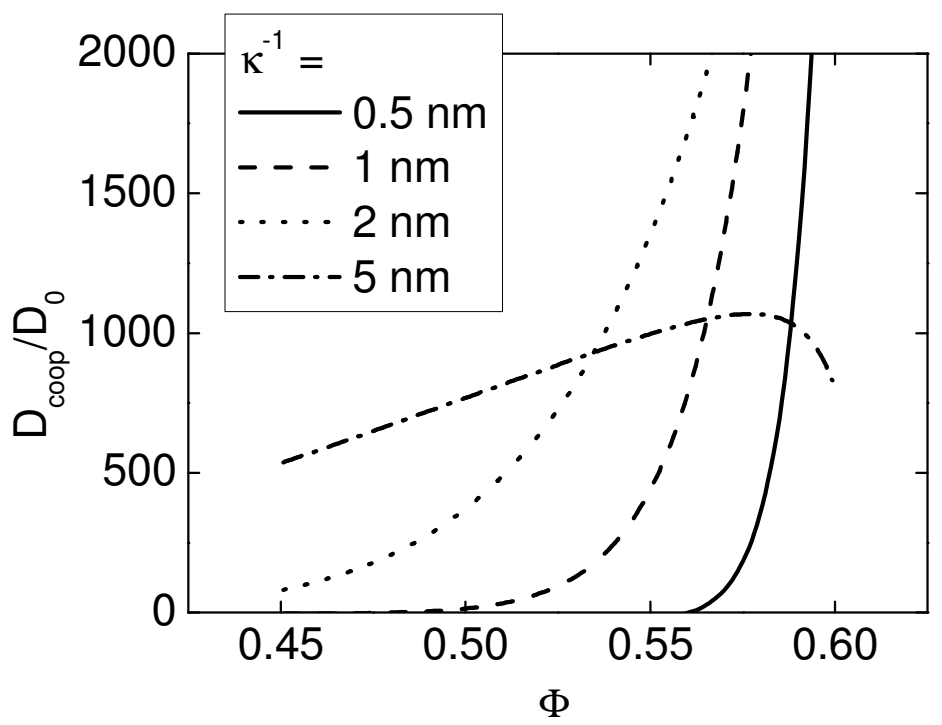

Fig. 9

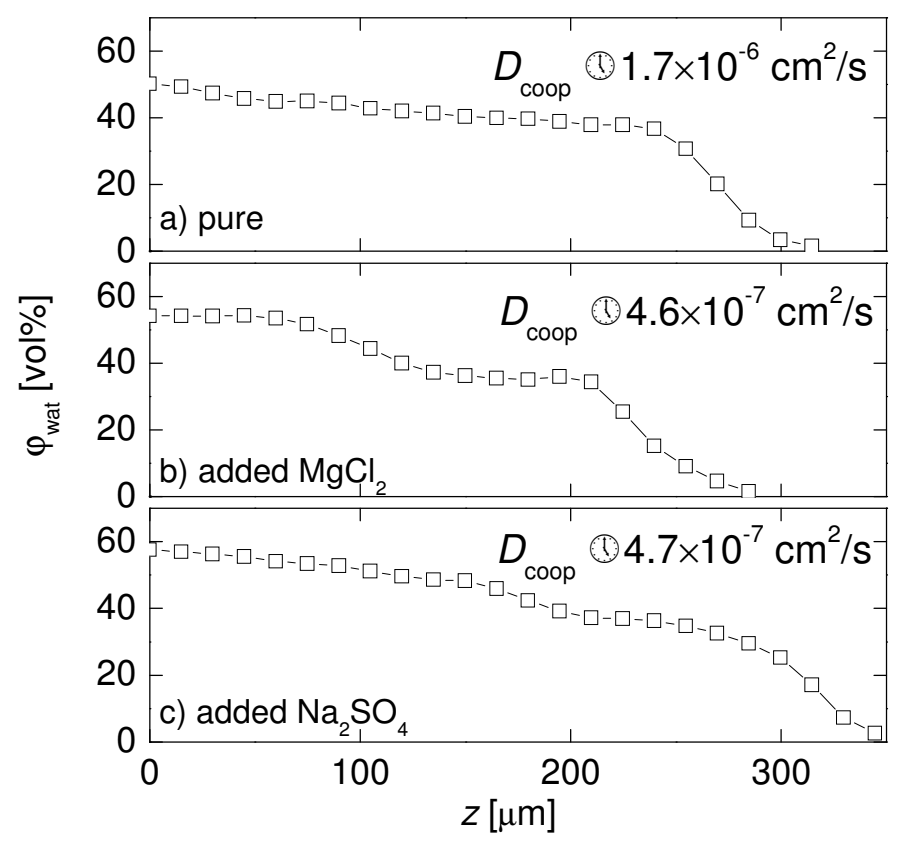


Published in Langmuir (2008) 24(14):7580-7589

Figure for TOC

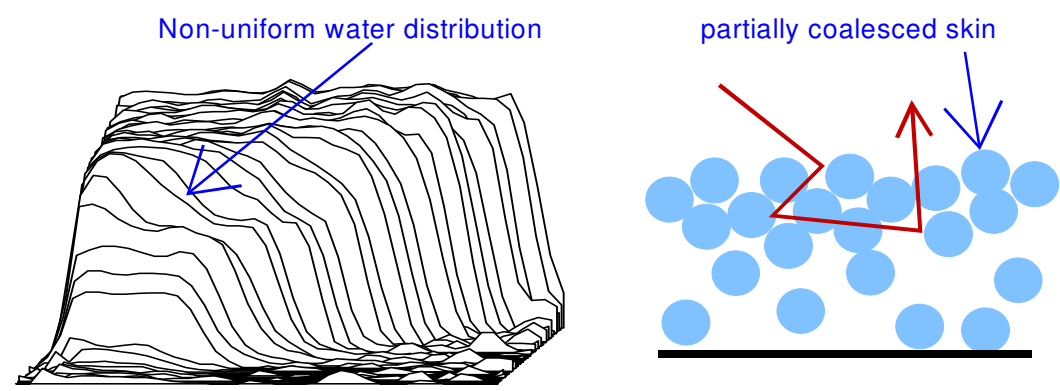

